\title{
TEORIA PER LA DETERMINAZIONE DEL CONTENUTO RADIOATTIVO DEI MINERALI
}

II.

Donato Palumbo

Considerazioni generali. - Nella prima parte di questo lavoro (1) alohiamo mostrato come il numero $F_{1}$ di tracce di particelle $\alpha$ prodotte per $\mathrm{cm}^{2}$ e $s$, in una emulsione nucleare, dalle particelle emesse dai nuclidi della specie $i^{m_{a}}$ appartenenti al contenuto radioattivo di un materiale sorrapposto alla emulsione, e soddisfacenti a talune condizioni geometriche che saranno imposte sotto, è dato da:

$$
F_{\mathrm{i}}=\frac{\lambda_{\mathrm{i}}^{\alpha}}{2} \int n_{\mathrm{i}} d s d v
$$

Questo integrale è esteso ad un dominio $S$ del piano $(v, s)$ determinato, unitamente alle

$$
n<1<1 \quad ; \quad s>0
$$

dalle limitazioni dovute alle caratteristiche geometriche imposte alle tracce.

Rimandiamo alla citata parte I per il significato dei simboli. Se la distribuzione dei nuclidi attivi della specie $i^{\text {ma }}$ può supporsi uniforme, ed il materiale omogeneo, si ha evidentemente:

$$
F_{\mathrm{i}}=\frac{\lambda_{\mathrm{i}}^{\alpha} n_{\mathrm{i}}}{2} S
$$

essendo $S$ l'area del dominio accennato.

Questa impostazione è stata usata nella I parte per determinare, in modo molto semplice, gli spettri integrali delle proiezioni orizzontali e rerticali, e delle lunghezze effettive delle tracce nell'emulsione, nell'ipotesi, generalmente ammessa, che il rapporto tra i percorsi di una particella a, di energia iniziale assegnata, in due mezzi sia indipendente dall'energia iniziale. Ora, come mostrano i lavori teorici e sperimentali su quest'ultimo argromento, tale ipotesi può rappresen- 
tare solo una prima approssimazione. Questo può rilevarsi, per la coppia aria cmulsione, confrontando la tabella percorso in emulsione-cner. gia, data da Rotblat, per le Ilford C2, e riportata da Landolt (2), con le curve percorso in aria/energia, riportate nella stessa opera. Il rapporto tra i percorsi risulta una funzione notevolmente crescente dell'energia iniziale. Vero è che esistono tuttora notevoli discrepanze intorno ai valori del percorso nell'emulsione, ma l'accennato aumento di $\mu_{,}$è nettamente al di fuori di tali discrepanze. Cosi, da una occhiata alla Tahella riportata da Lonchamp ('3), si nota che, per particelle a di $2.18 \mathrm{MeV}$ (corrispondenti ad un percorso in aria di 1.12 cmi, i percorsi in emulsione $C \Omega$, (prendendo gli errori indicati nel verso più sfavorevole), variano, per i diversi sperimentatori, tra 7.01 e 7.08 micron. Si arreblie cosi, per il rapporto $\mu_{.,}$tra il percorso in aria e quello nell'emulsione:

$$
1.582<\mu_{2}<1.600
$$

Analogamente, per le tracce date dal $T h C^{\prime},(8.57 \mathrm{~cm}$ d'aria), il percorso nell'emulsione varierehhe tra 46 e 47.8 micron, con che:

$$
1.793<\mu_{2}<1.863
$$

Come si vede, l'ipotesi che $u$ almeno limitatamente alla coppia aria emulsione, sia indipendente dall'energia iniziale, $\grave{e}$ certamente errata, ed in misura nettamente superiore ai possibili errori sperimentali. Prendendo, per i percorsi nell'emulsione, in corrispondenza alle di. verse energrie, i valori piu attendibili, si vede che, nel caso in esame, u varia con continuita da 1.550 circa, per le particelle aventi nell'emulsione percorso dell'ordine di 5 micron, ad un po' più di $1.80(1)$ per le $\alpha$ del $T h C^{\prime}$, come del resto $\dot{e}$ mostrato in un grafico che figura nel citato lavoro di Lonchamp.

Questi risultati sperimentali sono perfetlamente giustificati dalla teoria della determinazione del percorso delle particelle cariche in funzione dell'energia iniziale, hasata sulla formula di Bethe, che dà la perdita di energia delle particelle nella materia. Ora, la perdita di energia in un dato mezzo, e quindi il percorso, dipende principalmente dai potenziali di ionizzazione dei vari elettroni presenti nel mezzo attraversato. La variabilità di tali potenziali da mezzo a mezzo implica la non proporzionalita tra i cammini, cioè la non costanza del rapporto $u$. Nel caso particolare aria/emulsione, la variazione di u è intimamente connessa al fatto che, mentre l'aria è composta da 
atomi di basso numero atomico, gli elettroni responsabili del rallentamento delle particelle $u$ nell'emulsione appartengono, in massima parle, ad atomi relativamente pesanti $(A g, B r)$.

Un ampio studio sul problema della determinazione del percorso di particelle $\alpha$, di energia iniziale assegnata in un dato mezzo, è stato fatto da Mano $\left(^{4}\right.$. Sul problema della determinazione teorica del cocfficiente di frenamento delle particelle a nei vari mezzi, esistono ampi lavori fra i quali ricordiamo, oltre quello di Mano, quello di Bethe $(")$ e, più recenti, quelli di Webb $\left({ }^{6}\right)$ e Vigneron ( ${ }^{7}$ ).

Una breve trattazione dell'argomento, accompagnata da una ampia bibliografia, è fatta da Beiser $\left({ }^{8}\right)$. In tutti questi lavori è confermato l'aumento con l'energia del rapporto di frenamento delle particelle " nei mezzi pesanti; in particolare Vigneron sviluppa completamente, per le emulsioni $C 2$, dei calcoli numerici per la determinazione delle curve percorso/energia che potrebhero essere ripetuti per qualsiasi altro mezzo di nota composizione chimica. Notiamo che i coefficienti ıt non hanno un significato teorico diretto, né, come vedremo in sequito, grande ulilita pratica, dal momento che si abbandona l'ipotesi approssimala della loro indipendenza dall'energia iniziale della particella. Uniformandoci alla nomenclatura esistente, converri chiamare $u$ coefficiente di arresto integrale, riservando il nome di coefficiente relativo di arresto al rapporto $\frac{d \eta}{d \xi}=\frac{d \eta}{d E} / \frac{d \xi}{d E}$, dove 5, 川 sono i percorsi nei due mezzi di una particella $\alpha$ di energia iniziale E. Per i materiali terrestri, per i quali si utilizza comunemente l'autoradiografia, e'e da pensare che la relazione tra il percorso nel materiale e nell'emulsione sia, almeno qualitativamente, dello stesso tipo di quella che si ha tra il percorso in aria e quello nell'emulsione. Infatti, questi materiali sono solitamente ricchi di elementi, come $l^{\prime} O$ e il $S i$, relativamente legrgeri rispetto all' $A g$ e $B r$. La crescenza del rapporto dei percorsi con l'enerwia sarà però per questi materiali meno pronunziata che per la coppia aria/emulsione.

Il problema dell'equilibrio delle famiglie radioattive. - L'aver trascurato l'accennata dipendenza del rapporto tra i percorsi dall'energia iniziale, porta ad errori sensibili sulle formule che danno grli spettri integrali se i percorsi $R_{\mathrm{i}}$ nell'emulsione dei vari gruppi di particelle us vengono dedolti dai corrispondenti percorsi in aria mediante il coefficiente l!: assunto come costante. Errori meno gravi, ma in taluni casi non trascurabili, si hanno assumendo per i percorsi nell'e- 
mulsione dei vari gruppi i valori corretti, ma continuando a ritenere costante il rapporto $\frac{d \eta}{d \vdots}$. Questi ultimi errori sono trascurabili quando si utilizzano le formule che danno rrli spettri integrali solo su piccoli intervalli di lunghezza, cioe praticamente, quando si ritiene sufficiente l'analisi delle sole tracce lunghe ("). Ora, quest'analisi ¿̀ sufficiente per la determinazione del rapporto tra le concentrazioni di $U$ e $T h$ qualora questi due elementi si possano supporre in equilibrio secolare con i loro discendenti; qualora cioè, l'età di formazione dei materiali esaminati sia superiore a $10^{5}$ a ed il minerale non abbia subito successivamente differenziazioni chimico-fisiche. Ma non sempre si può fare questa ipotesi, e non mancano Autori $\left({ }^{10}\right)$ che ritengrono imprudente l'applicazione delle formule semplificate, valide solo nel caso dell'equilibrio. Nella famigrlia dell'U, la mancanza dell'equilibrio, per difetto di età, può portare ad un eccesso dei primi elementi della famiglia rispetto ai successivi, nel caso in cui l'elemento inizialmente presente nel minerale sia stato solo l' $U$; ad un difelto di $U$ rispetto agli elementi successivi della famiglia, nel caso in cui siano intervenuti, in epoche non remote, processi di differenziazione che abhiano favorito qualcuno dei discendenti a vita lunga deil'U (lo, Ra). L'esistenza di tali processi è sugrgerita da Rankama e Sahama ("); in hase all'aumento del rapporto $T h U$ osservato da Senftle e Keevil, nel passaggio dalle rocce innee acide a quelle innce intermedie. Questi A.A. spiegano tali variazioni con l'ipotesi che i processi di cristallizzazione abbiano favorito il $T h$ rispello all' $U$. E poiché l'lo ì isotopo del $T h$, c'e da pensare che, come risultato del processo di cristallizzazione, l'lo, e quindi piuttosto rapidanente, e per un tempo dell'ordine di $10^{\overline{ }}$ a, gli elementi ad esso successivi vengano a trovarsi con una concentrazione superiore a quella che competerebbe loro se fossero in equilibrio con l' $U$ presente. Analoghi fenomeni potrebhero aversi per il $R a$, anzi, in mancanza di altre ragioni, questi fenomeni potrebbero rendere conto del regolare aumento di attività delle lave Vesuviane con la data delleruzione, rilevato da Joly e confermato da Pachelet, Ferretti e Ippolito ${ }^{10 \%}$. Altra catsa di errore, come ha fatto notare a proposito di altri fenomeni il Professor lmbò (*), può essere dovuta alla perdita di $R_{n}$ per dilfusione da parte del campione esposto, durante l'esposizione. Da quanto detto risulta evidente l'opportunità di spingrere le misure fino alle tracce più corte,

(*) Comunicazione al Congresso dell'A.I.G. Roma, giugno 1953. 
essendo le a emesse dai primi elementi della famiglia dell' $U$, di energia relativamente bassa rispetto a quelle emesse dagli elementi successivi.

Come mostreremo iu seguito con qualche esempio numerico, l'applicazione delle formule basate sull'ipotesi della proporzionalità dei percorsi delle $\ell$ nei diversi mezzi attraversati può portare ad errori cbe influiscono sensibilmente sulla determinazione del rapporto $U / T h$, e clie possono falsare in misura notevole le deduzioni sull'equilibrio della famiglia dell' $U$.

Oltre alla non proporzionalità fra i cammini, le principali fonti di errore possono essere la non omogreneità del materiale osservato, e gli errori di osservazione e di misura. Per quanto riguarda questi ultimi, il Vigneron (') fa notare che le misure delle proiezioni orizzontali delle tracce, se correttamente eseguite, possono essere fatte con un errore inferiore allo $0,25 \%$. Per quanto riguarda la omogeneità, daremo nel seguito una condizione necessaria. Supporremo che il numero delle tracce misurate sia sufficientemente grande perché si possano trascurare gli errori statistici. Infine, in taluni casi, per evitare azioni chimiche del materiale sull'emulsione, non è possibile sovrapporre direttamente quest'ultimo all'emulsione, ma occorre lasciare uno strato d'aria o di altro mezzo. Anche in questo caso, saranno trovate nel seguito le formule che danno lo spettro integrale.

Determinazione degli spettri integrali. - Indichiamo con 5,11 i cammini nell'emulsione e nella materia di una particella a di energia iniziale $E$. Si avrà

$$
1:=g(\eta)=h(\xi)
$$

con $h, g$ funzioni monotone crescenti dei loro argomenti. La [3] implica tra $\mathrm{i}$ percorsi di particelle isoenergetiche in emulsione ed in materia la relazione

$$
\eta=l(\xi)
$$

essendo $l$ una funzione monotona crescente di $\div$.

Consideriamo, come nella parte $I$, un minerale limitato da una superficic piana sovrapposta ad una emulsione nucleare. Siano $R_{\text {i }}$, $R_{i}^{\prime}$ i percorsi in emulsione e minerale rispettivamente, dell' ${ }^{m o}$ oruppo di particelle $a$; sari, per la [4]:

$$
R^{\prime}{ }_{i}=l\left(R_{\mathrm{i}}\right)
$$


Detto $b^{\prime}$ il cammino residuo nell'emulsione, a quello nel minerale, si arri, per la [3]:

$$
g\left(R^{\prime}{ }_{i}-a\right)=h\left(b^{\prime}\right)
$$

da cui, essendo $g, h$ monotone, e per la [4]:

$$
a=l\left(R_{i}\right)-l\left(b^{\prime}\right)
$$

Se $y$ è l'angolo formato dalla direzione di emissione della particella $\alpha$ con la normale al piano di separazione tra il minerale $e$ l'emulsione, la profondita s da cui proviene la particella sarà data da $s=a \cos \vartheta=a v$, da cui :

$$
s=v\left[l\left(R_{\mathrm{i}}\right)-l\left(b^{\prime}\right)\right]
$$

Indicando con $\phi_{1}(r), \psi_{i}(r), f_{1}(r)$ il numero di tracce aventi nella lastra proiezione orizzontale, verticale o percorso maggiori di una quantità assegnata $r$, per il dominio $S$ definito dalle $[1],\left[1^{\prime}\right]$, si avranno le limitazioni :

$$
\begin{aligned}
0 \leq s \leq v\left[l\left(R_{\mathrm{i}}\right)-l\left(\frac{r}{l^{r}}\right)\right] \\
0 \leq s \leq v\left[l\left(R_{\mathrm{i}}\right)-l\left(\frac{r}{v}\right)\right] \\
0 \leq s \leq v\left[l\left(R_{\mathrm{i}}\right)-l(r)\right]
\end{aligned}
$$

che, unitamente alle [2], determinano $S$.

Si può intanto dimostrare che lo spettro integrale delle proiezioni verticali coincide con quello delle orizzontali, indipendentemente dalla forma della funzione $l(E)$. Si ha infatti per la [6]:

$$
\varphi_{\mathrm{i}}(r)=\frac{\boldsymbol{\kappa}_{\mathrm{i}}^{\alpha} n_{\mathrm{i}}}{2} \int_{0}^{v^{\circ}}\left[l\left(R_{\mathrm{i}}\right)-l\left(\frac{r}{\sqrt{1-v^{2}}}\right)\right] v d_{v}
$$

essendo $v^{\prime \prime}=\sqrt{1-\left(\frac{r}{R_{\mathrm{i}}}\right)^{2}}$ l'unica radice positiva $l\left(R_{\mathrm{i}}\right)=l\left(\frac{r}{\sqrt{1-v^{\alpha}}}\right)$ e, per la $\left[6^{\prime}\right]$ :

$$
\eta_{i}(r)=\frac{\lambda_{\mathrm{i}} n_{\mathrm{i}}}{\dot{L}} \int_{\frac{r}{R}}^{1}\left[l\left(R_{\mathrm{i}}\right)-l\left(\frac{r}{v}\right)\right] v d v
$$


Ora, il primo integrale con il cambiamento di variabile $t=\sqrt{l-v^{\ddot{2}}}$, $\therefore$ trasforma appunto nell'ultimo. si ha quindi, qualunque sia $i(\xi)$,

$$
\begin{array}{r}
\varphi_{\mathrm{i}}(r)=\psi_{\mathrm{i}}(r)=\frac{\lambda_{\mathrm{i}}^{u} n_{\mathrm{i}}}{2} \int_{\frac{r}{K_{\mathrm{i}}}}^{\int}\left[l\left(R_{\mathrm{i}}\right)-l\left(\frac{r}{v}\right)\right] v d v \\
\left(r<R_{\mathrm{i}}\right) ; \quad \varphi_{\mathrm{i}}(r)=\psi_{\mathrm{i}}(r)=0, \quad\left(r>R_{\mathrm{i}}\right) .
\end{array}
$$

E, per la [6]:

$$
f_{\mathrm{i}}(r)=\frac{i_{\mathrm{i}}^{\alpha} n_{\mathrm{i}}}{4}\left[l\left(R_{\mathrm{i}}\right)-l(r)\right] \quad r \leq R_{\mathrm{i}} ; \quad f_{\mathrm{i}}(r)=0,(r>R) .
$$

Supponiamo ora che le due superfici piane e parallele che limitano il materiale e l'emulsione siano separate da uno strato non contenente nuclidi radioattivi.

Facciamo per semplicità l'ipotesi che il coefficiente di arresto di tale mezzo sia proporzionale a quello del materiale. Sia $d$ il suo spessore espresso in unità equivalenti di spessore del materiale. La [6] diventa allora:

$$
0 \leq s \leq\left[l\left(R_{\mathrm{i}}\right)-l\left(\frac{r}{\sqrt{1-v^{\bar{c}}}}\right)\right] v-d \geq 0
$$

indicando con $v_{1}, v_{2}$ i due valori di $v$ compresi tra 0 e $l$ che annullazno il terzo membro della [9], si arrà evidentemente per il numero di tracce aventi proiezione orizzontale $r$ :

$$
\Phi_{\mathrm{i}}(r)=\frac{\lambda_{i}^{\tilde{n}} n_{\mathrm{i}}}{r}\left\{\int_{v_{1}}^{v_{2}}\left[l\left(R_{\mathrm{i}}\right)-l\left(\frac{r}{\gamma\left(1-v^{2}\right.}\right)\right] v d v-\left(v_{\mathrm{a}}-v_{1}\right) d\right\}
$$

La determinazione di $\Phi_{i}(r)$ esige evidentemente la conoscenza della funzione $l(\xi)$; se però $d$ è molto piccolo, cosa che in pratica può sempre realizzarsi, per valori di $r$ non molto vicini ad $R_{\mathrm{i}}, v_{1}$ e $v_{3}$ differiranno poco dai valori che avrebbero per $d=0$, cioè da 0 e $\sqrt{1-\left(\frac{r}{R_{\mathrm{i}}}\right)^{2}}$ rispeltivamente.

E, notando che nella [10] l'integrando si annulla ai limiti, e quindi lintegrale risente pochissimo piccole variazioni di $v_{1}$ e $v_{2}$, si 
avrà con buona approssimazione:

$$
\Phi_{\mathrm{i}}(r) \simeq \varphi_{\mathrm{i}}(r)-\frac{\tilde{\lambda_{\mathrm{i}}} \boldsymbol{n}_{\mathrm{i}}}{2} \sqrt{1-\left(\frac{r}{k_{\mathrm{i}}}\right)^{2}} d
$$

In modo analogo si procederà per la definizione, determinazione e approssimazione di $\Psi_{\mathrm{i}}(r), F_{\mathrm{i}}(r)$. Si trova sotto le stesse ipotesi fatte a proposito di [11]:

$$
\Psi_{\mathrm{i}}(r) \approx \psi_{\mathrm{i}}(r)-\frac{\lambda_{\mathrm{i}}^{\alpha} n_{\mathrm{i}}}{2}\left(1-\frac{r}{R_{1}}\right) d
$$

Le [11] [11'] mostrano come, nel caso del mezzo interposto, non oi abbia più la coincidenza tra gli spettri integrali delle proiezioni orizzontali e di quelle verticali. Infine, nel caso considerato da Imbò e Casertano $\left({ }^{12}\right)$, in cui lo spessore $h$ del minerale sia minore di $l\left(R_{1}\right)$, al dominio $S$ va imposta l'ulteriore limitazione $s \leqslant h$.

In questo caso, lo spettro $\varphi_{1}(r)$ delle proiezioni orizzontali, se la

$$
v\left[l\left(R_{\mathrm{i}}\right)-l\left(\frac{r}{\sqrt{1-v^{2}}}\right)\right]=-h
$$

ammette due radici $v_{1}, v$, comprese tra 0 e $v^{o}=\sqrt{1-\left(\frac{r}{R_{\mathrm{i}}}\right)^{2}}$, è dato

$$
\begin{gathered}
\left.\bar{\varphi}_{\mathrm{i}}(r)=\varphi_{\mathrm{i}}(r)-\frac{\lambda_{\mathrm{i}}^{u} n_{\mathrm{i}}}{2} \int_{v_{1}}^{v_{\mathrm{a}}}\left[l\left(R_{\mathrm{i}}\right)-l\left(\frac{r}{\sqrt{1-v^{\alpha}}}\right)\right] v d v-h\left(v_{\mathrm{o}}-v_{\mathrm{i}}\right)\right\} \\
r<R_{\mathrm{i}} . \quad\left[7^{\prime}\right]
\end{gathered}
$$

Se non esistono le radici $v_{1}$, $v$, comprese tra i limiti indicati, per $\bar{\varphi}_{\mathrm{i}}(r)$ continua ad essere valida la [7].

Analogamente, se esiste una radice $v_{1}$, soddisfacente alla limitazione $\frac{r}{R_{1}} \leq: v, \leq 1$, della

$$
v\left[l\left(R_{\mathrm{i}}\right)-l\left(\frac{r}{v}\right)\right]=h
$$

si avrà per lo spettro delle proiezioni orizzontali:

$$
\bar{\psi}_{\mathrm{i}}(r)=\frac{\wedge_{\mathrm{i}}^{\alpha} n_{\mathrm{i}}}{2}\left\{\int_{\substack{r \\ \bar{R}_{\mathrm{i}}}}\left[l\left(R_{\mathrm{i}}\right)-l\left(\frac{r}{v}\right)\right] v d v+h\left(1-v_{\mathrm{i}}\right)\right\} \quad r<R_{\mathrm{i}}
$$

nel caso contrario $\bar{\psi}_{\mathfrak{i}}(r)$ è dato ancora dalla [7]. 
Per lo spettro delle lunghezze effettive, se $v_{\underline{1}}=\frac{h}{l\left(R_{\mathrm{i}}\right)-l(r)}<1$,

$$
\overline{f_{\mathrm{i}}}(r)=\frac{\lambda_{\mathrm{i}}^{\alpha} n_{\mathrm{i}}}{2} h\left(1-\frac{v_{\mathrm{i}}}{2}\right), \quad r \leq R_{\mathrm{i}}
$$

mentre continuerà ad esser valida la $[8]$ se $h>l\left(R_{1}\right)-l(r)$.

Ricerca dolla funzione $l(\xi)$. - Le maggiori difficoltà risiedono nella determinazione della prima funzione $l(E)$. Come primo tentativo si è posto:

$$
l(\xi)=b\left(\xi+k \xi^{2}\right)+c
$$

essendo $k, b, c$ tre costanti da determinare. Ponendo la [12] nelle [7], [8] si ha:

$$
\varphi_{\mathrm{i}}(r)=\eta_{\mathrm{i}}(r)=\frac{\lambda_{\mathrm{i}}^{\alpha} n_{\mathrm{i}}}{4} b\left\{\frac{\left(R_{\mathrm{i}}-r\right)^{2}}{R_{\mathrm{i}}}+k\left[R_{\mathrm{i}}^{2}-2 r^{2} \lg _{\mathrm{e}} R_{\mathrm{i}}+r^{2} \lg _{\mathrm{e}} \frac{r^{2}}{e}\right]\right\}
$$

$$
\left.f_{\mathrm{i}}(r)=\frac{\lambda_{i}^{\alpha} n_{\mathrm{i}}}{4} b\left[\left(R_{\mathrm{i}}-r\right)+k_{(} \boldsymbol{R}_{\mathrm{i}}=-r^{2}\right)\right]
$$

Le [13], [13'] si riducono ovviamente, per $k=0$, alle ben note [6], [4] della parte $I$, che indicheremo in seguito con $\left(i^{\circ}(r), f_{i}^{\circ}(r)\right.$. Indicheremo con $\varphi_{i}^{\prime}(r), f_{i}^{\prime}(r)$ i termini di [13], [13'] che hanno coefficiente $k$.

Come mostrano queste formule, e come si vedrà meglio in seguito, l'unico coefficiente che occorre conoscere per trattare il problema dell'equilibrio e del rapporto tra le concentrazioni dei capostipiti delle varie famiglie radioattive è $k$. La determinazione di $k$ pui esser tentata empiricamente qualora si conoscano un numero sufficiente di coppie di valori $\zeta, \|$; questo potreblue farsi, p. es., per la coppia aria/emulsione. Si trovano cosi, utilizzando parte dei dati riportati da Lonchamp $\left({ }^{3}\right)$ per le $C 2$ Ilford, e per $\xi$ variabile tra $i$ e 47 micron, $k=(2 \div 3)$. $10^{3}$. Un valore legrermente superiore si trova utilizzando $i$ dati di Rothlatt. Per $i$ percorsi in aria si sono usati i dati riportati nella citata opera del Landolt.

Però l'accordo tra i dati sperimentali e la [12] non è molto buono, sia per l'inadeguatezza di quest'ultima, sia forse per la imprecisione dei dati sperimentali. Inoltre la determinazione a priori di $k$ 
riuscirebbe più difficile nei casi praticamente occorrenti di materiali per i quali non si disponga di sufficienti dati. 11 parametro $k$ dovreb. he considerarsi come una costante da determinare a posteriori in modo da ottenere il migliore accordo possibile tra lo spettro leorico e quello osservato. ì prevedibile che per i materiali terrestri comuni (SiO..), i valori di $k$ saranno circa la mela che per l'aria. Un metodo mighiore per tentare una approssimazione della funzione $n=l(\varepsilon)$ consiste, come fa giustamente rilevare, per un caso analogo, (calcolo del cammino nell'enulsione in funzione dell'energia iniziale), il Vi. gneron, nell'utilizzazione della formula di Bethe. Si ha:

$$
-\frac{d E}{d x}=\frac{16 x^{2} e^{4}}{m v^{2}} N Z\left[\lg =2 m v^{2}-\lg =I-\lg _{\mathrm{c}}(1--\beta 2)-\beta^{2} \mid\right.
$$

ed analogamente:

$$
-\frac{d E}{d y}=\frac{16 \pi^{2} e^{4}}{m v^{2}} N^{\prime} Z^{\prime}\left[\lg _{e} 2 m v^{\alpha}-\lg _{e} I^{\prime}-\lg _{\mathrm{c}}\left(1-\beta^{2}\right)-\beta^{2}\right]
$$

Per il significato dei simboli, rimandiamo al citato lavoro del Vigneron. Da queste due formule con facile ragionamento si deduce:

$$
l^{\prime}(\xi)=\frac{N Z}{N^{\prime} Z^{\prime}} \frac{\lg _{10} E+6-\lg _{10} \frac{M}{4 m}-\lg _{10} I}{\lg _{10} E+6-\lg _{10} \frac{\boldsymbol{M}}{4 m}-\lg _{10} I^{\prime}}
$$

In questa formula, in cui si sono trascurate le correzioni relativistiche, (termini in $\beta^{2}$ ), $E$ è l'energria cinetica iniziale della particella $\alpha$ misurala in $M e V, M$ ed $m$ la massa di riposo della particella r e dell'elettrone, I ed I' i potenziali medi di ionizzazione degrli elettroni responsabili del rallentamento nell'emulsione e nel materiale: $N Z, N^{\prime} Z^{\prime}$ il numero di elettroni responsabili del rallentamento per $\mathrm{cm}^{3}$ di emulsione e di materiale rispettivamente. Per la definizione esalta di $N Z$ e di $I$ rimandiamo al lavoro del Vigneron. Si noti che $N Z$ ed $I$ dipendono dall'energia perché al decrescere di questa gli clettroni più fortemente legati, cioè particolarmente quelli dei livelli $K$. $L$ degli atomi pesanti, cessano di contribuire al rallentamento.

Servendoci della [14] abbiamo calcolato i valori del coefficiente $b_{i}$ della [12] per la coppia $\mathrm{SiO}_{2} / \mathrm{cmulsione,} \mathrm{con} \mathrm{il} \mathrm{risultato} \mathrm{sopra} \mathrm{accen-}$ nato. Però, anche in questo caso, la [12] non è in buon accordo con 
la [1+]. Ln migliore accordo si trova assumento $l^{\prime}(5)$ della forma:

$$
l^{\prime}(\xi)=b\left(1-\frac{k}{\xi}\right)
$$

con cine:

$$
l(\xi)=b\left(\xi-k \lg _{\mathrm{e}} \xi\right)+c
$$

il valore di $k$ calcolato sia in base alla [14] per $\mathrm{SiO}_{22}$ che in hase ai risultati di Webl, Bethe e Livingstone, per $A l$ risulta circa 1,2 per energie iniziali delle a comprese fra 3 ed 8,5 $\mathrm{MeV}$. Ponendo la [15'] nella [7] si trova:

$$
\varphi_{\mathrm{i}}(r)=\frac{{\vartheta_{\mathrm{i}}^{\alpha}}^{\alpha} n_{\mathrm{i}}}{4} \delta\left\{\frac{\left(R_{\mathrm{i}}-r\right)^{2}}{R_{\mathrm{i}}}-k\left[l_{\mathrm{g}} R_{\mathrm{t}}-\left(\frac{1}{2}+\lg _{\mathrm{e}} r\right)+\frac{r^{2}}{2 R_{\mathrm{i}}{ }^{2}}\right]\right\}
$$

Indicheremo con $p_{i}^{\prime}(r)$ il termine della [16] che ha coefficiente- $k$.

Spettro integrale di una famiglia in equilibrio. - Lo spettro integrale di una famiglia radioattiva è evidentemente dato dalle:

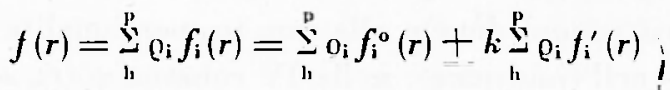

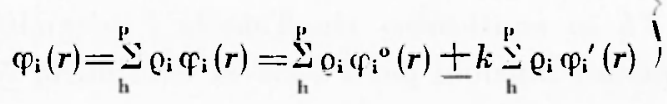

con le ovvie relazioni tra gli $n_{1}$, e dove $\rho_{i}$ è un coefficiente dovuto alle eventuali biforcazioni. Le $f, \varphi$ sono date dalle [7], [8] o da [16]. Il calcolo numerico delle [17], [18] viene semplificato dalla possibilita di spezzare le sommatorie, sia nel caso già considerato nella parte $I$, sia nei casi in cui la $l(5)$ abbia le forme [12] o [15'], (lo slesso accade per le altre forme di $l(\vdots)$ di cui abhiamo tentato l'applicazione, e qui non riportate), in un certo numero di sommatorie, da ciascuna delle quali è possibile mettere in evidenza i fattori dipendenti da $r$, sicché le sommatorie risultano indipendenti da questa unica variabile, ma funzioni, molto semplici, delle sole costanti $R_{i}$ e degcli eventuali rapporti di biforcazione.

Nell'ipotesi della validiti della [12] o della [15], occorre calcolare le

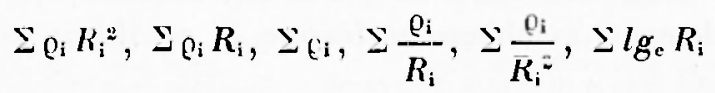


tutle estese da $h$ a $p$, con $h=\mathrm{I}, \ldots, p$, dove $p$ ¿̀ il numero di specie di elementi radioattivi a della famiglia considerata. Per mezzo di queste sono state costruite le Tabelle I e II. La I è stata calcolata nell'ipotesi che valga la $[12]$; i percorsi nell'emulsione delle particelle $a$ sono stati tratti, in parte, del citato lavoro di Lonchamp, in parte interpolati assumendo come valori delle energie delle particelle a quelli riportati nel citato volume del Landolt. In entrambe le Tabelle si ̀̀ assunto, per il $T h C^{\prime}, \varrho .=2 / 3$; non si è tenuto conto di altre biforcazioni.

La Tabella II è stata calcolata nell'ipotesi che sia valida la [15]. I percorsi delle particelle u nell'emulsione sono stati dedotti dai dati di Rotblatt, usando per le energie, i valori riportati da M. JoliotCurie ${ }^{(13}$ ). In ciascuna Tabella sono riportati, nella I colonna $r$ espresso in micron, (per semplicita sono dati $i$ valori $d i$ (" solo in corrispondenza ai valori di $r$ coincidenti con i vari $R_{\mathrm{i}}$, il che $\dot{e}$ sufficiente per l'interprelazione degli spettri osservati sperimentalmentel; nella II colonna l'elemento le cui particelle hanno percorso nell'enulsione $R_{\mathrm{i}}=r$; nella III i valori $\varphi^{\circ}(r)$ per la famiglia del $T h$ in equilibrio, (cioè lo spettro integrale prodotto da questa famiglia nel caso in cui si possano trascurare le correzioni dovute alla non proporzionalita tra i percorsi nella materia e nell'emulsione); nella IV colonna $q^{\prime}(r)$, sempre per la famiglia del $T h$ in equilibrio; (in Tabella $\left.I \quad f^{\prime}(r) / 1000\right)$; nella V e VI le corrispondenti funzioni per l'U238 in equilibrio. Nella VII ed VIII le analoghe per l'lo in equilibrio con gli elementi ad esso successivi; analogamente nella IX e $\mathrm{X}$ per il $R a$ in equilibrio. Nella Tabella II, la colonna $\mathrm{VI}^{\prime}$ contiene alcuni dati relativi allo spettro integrale della famiglia dell' $U 238$ in equilibrio, calcolati nella ipotesi che i percorsi nella materia e nell'emulsione siano proporzionali tra loro, e l'ultimo proporzionale al percorso in aria secondo il coefficiente 1.750 suggerito da Yagoda ed usato da Buttlar ed Houtermans, ed assumendo per $\mathrm{i}$ percorsi in aria $\mathrm{i}$ valori riportati da $\mathrm{M}$. Joilot-Curie.

Nella Tabella II sono riportate le concentrazioni di $U 238$ e $T h$, $\left(C_{\mathrm{U}}, C_{\mathrm{Th}}\right)$, espresse in $\mathrm{gr} / \mathrm{cm}^{3}$ che producono, per $\mathrm{cm}^{-}$e $s$ gli spettri integrali calcolati.

Le concentrazioni di lo e Ra che danno luogo agli spettri delle famiglie che traggono origine da questi due elementi sono quelle stesse che questi elementi avrebbero se fossero in equilibrio con la concentrazione $C_{:}$di $U$. 
Tabella I

\begin{tabular}{|c|c|c|}
\hline I & II & \\
\hline 5 & - & \\
\hline 16 & - & \\
\hline 16,3 & & Th \\
\hline 18,8 & Io & \\
\hline 19 & UII & \\
\hline 19,5 & $\mathbf{R a}$ & \\
\hline 22 & Po & \\
\hline 22,9 & & RaTl \\
\hline 23,2 & Rn & \\
\hline 24,6 & & ThX \\
\hline 26,6 & $\operatorname{Ra} \Lambda$ & \\
\hline 27 & & ThC \\
\hline 28,7 & & $\mathrm{Tn}$ \\
\hline 31,8 & & ThA \\
\hline 38,4 & $\mathrm{RaC}$ & \\
\hline 47,2 & & ThC' \\
\hline
\end{tabular}

$\begin{array}{cc}\text { III } & \text { IV } \\ 1.000 & 38,88 \\ 313,8 & 17,13 \\ 302,5 & 16,62 \\ 220 & 12,68 \\ 214,3 & 12,39 \\ 200,6 & 11,68 \\ 114,3 & 8,59 \\ 129,1 & 7,68 \\ 124,4 & 7,39 \\ 105,9 & 6,19 \\ 86,69 & 6,80 \\ 83,99 & 4,56 \\ 46,64 & 3,68 \\ 31,66 & 2,51 \\ 10,31 & 0,87 \\ 0 & 0\end{array}$

$\mathrm{J}$
1.000
201,08
190,44
120,64
116,48
106,90
73,83
59,91
57,07
45,19
31,98
29,86
21,61
9,90
0
0

II
34,21
10,39
9,95
6,92
$6,7.4$
6,28
5,26
3,85
3,67
2,99
2,15
2,01
1,48
0,67
0
0

VII
811,45
196,71
186,69
120,55

VIII
29,10
10,24
9,83
6,91

\begin{tabular}{cr} 
IX & \multicolumn{1}{c}{ X } \\
751,64 & $27,0:$ \\
193,08 & 10,10 \\
183,91 & 9,72 \\
120,55 & 6,91 \\
116,48 & 6,73
\end{tabular}

\section{TABELLA II}

\begin{tabular}{|c|c|c|c|c|c|}
\hline I & II & III & IV & $V$ & VI \\
\hline 5 & 一 & 10.000 & 638.9 & 10.000 & 717.1 \\
\hline 10 & 一 & 6.211 & 301 & 5.425 & 291.5 \\
\hline 16.2 & Th & 2.993 & 113.8 & 1.976 & 80.64 \\
\hline 16.3 & $\mathbf{U}$ & 2.965 & 111.9 & 1.941 & 78.71 \\
\hline 18.6 & Io & 2.156 & 78.85 & 1.273 & 47.05 \\
\hline 19.4 & UII & 1.917 & 67.5 .1 & 1.106 & 39.64 \\
\hline 19.6 & $\mathbf{R a}$ & 1.821 & 63.23 & 1.076 & 37.98 \\
\hline 22.5 & Po & 1.182 & 36.45 & 617 & 21.68 \\
\hline 23.2 & RaTh & 1.060 & 31.9 & 575.5 & 18.12 \\
\hline 23.7 & $\mathbf{R n}$ & 979 & 29.01 & 575.5 & 16.52 \\
\hline 26.9 & $\operatorname{RaA}$ & 812 & 23.21 & 439.5 & 13.21 \\
\hline 27.3 & ThC & 594.6 & 16.07 & 304.6 & 8.89 \\
\hline 28.7 & $\mathrm{Tn}$ & 559.6 & 14.95 & 284 & 8.25 \\
\hline 32 & ThA & .154 .5 & 9.97 & 216.2 & 6.20 \\
\hline 38.5 & $\mathbf{R a C}^{\prime}$ & 286.7 & 6.96 & 95.57 & 2.77 \\
\hline 47 & $\mathrm{ThC}^{\prime}$ & 92.0 & 1.99 & 0 & 0 \\
\hline 24.9 & ThX & 0 & 0 & 0 & 0 \\
\hline
\end{tabular}

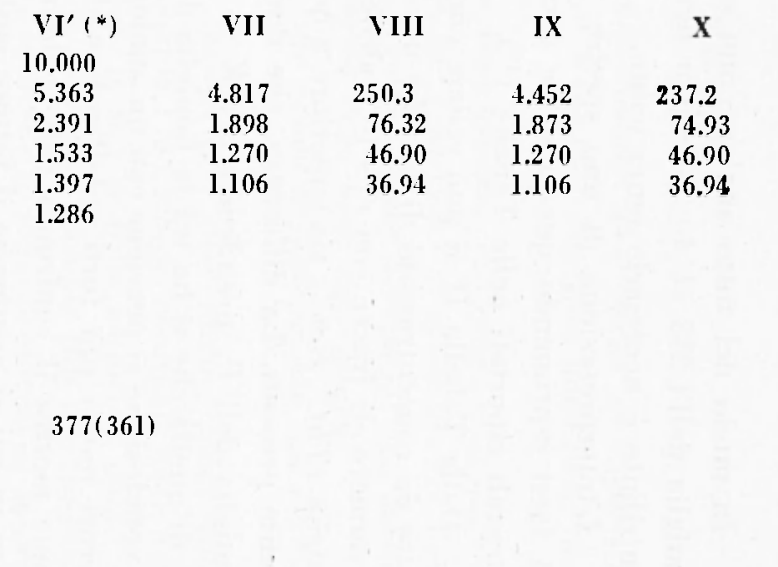

$C_{\mathrm{U}}=\frac{1}{b} 1.96 .10^{\circ} \cdot \mathrm{gr} / \mathrm{cm}^{3} \quad ; \quad C_{\mathrm{Th}}=\frac{1}{b} 6.08 .10^{-2} \mathrm{gr} / \mathrm{cm}^{3}$

(*) I dati riportati in questa colonna si riferiscono ai valori di $r$ uguali al percorso nell'emulsione delle particelle $\alpha$ dell'elemento della II colonna dedótto dal percorso in aria mediante il coefficiente 1.750; fa eccezione il dato in parentesi corrispon. dente a 26.9 micron. 
In modo del tutto analogo può essere calcolato lo spettro della famiglia dell' $U 235$ ( $U A c$ ), che non figura in queste tabelle, e del cui contributo è necessario tener conto.

L'interpretazione di uno spettro, ottenuto sperimentalmente potrà farsi esprimendo questo come combinazione lineare degli spettri integrali riportati nelle Tabelle $\left({ }^{14}\right)$.

Dalla Tabella II si può vedere come, nel caso di uno speltro prodotto da concentrazioni di $U$ e $T h$ uguali, to proporzionalis, a $C_{v}, C_{\mathrm{Th}}$, il numero di tracce con $r>10$, calcolato in base al numero di tracce lunghe (Th $C^{\prime}, R a C^{\prime}$, sia superiore a quello delle tracce corte effettivamente presenti. La differenza, che dovrebhe essere imputata alla sola famiglia dell' $L$, prendendo per $K$ il valore indicato sopra, è circa i $2 / 3$ di quella che si ha tra la famiglia dell' $U$ e quella dell' Io. Ad uguale conclusione si perviene con un analogo calcolo basato sulla Tabella $I$. Errori molto più forti, (dell'ordine del $20 \%$ del numero di tracce), come mostra il confronto delle colonne V, VI, VI', si avrehbero nel caso in cui si assumesse il fattore costante $u^{2}=1.750$.

Palermo - Istituto di Fisica dell'Università - Ottobre 1953.

\section{RIASSUNTO}

Facendo seguito ad una precedente nota, vengono determinati gli spettri inlegrali delle proiezioni orizzontali, verticali e delle lunghezze effettive delle tracce di particelle a emesse dai nuclidi attivi contenuti in un materiale sovrapposto ad una emulsione nucleare, nell'ipotesi generale che tra il percorso $\eta$ nella materia ed il percorso $\xi$ nell emulsione di una particella a di data energia iniziale sussista una relazione $\eta=1(z)$. Si dimostra che, supponendo il materiale omogeneo, di spessore superiore al percorso in esso delle particelle $u$, e direttamente sovrapposto alla emulsione, lo spettro delle proiezioni orizzontali coincide con quello delle verticoli.

Si considerano $i$ casi in cui alcune delle ipotesi sopra dichiarate non si siano verificate.

Si eseguono i calcoli numerici, dopo aver fatte alcune ipotesi sulla forma della funzione $1(\xi)$, nolla quale le costanti vengono calcolate in base alla teoria di Bethe, o partendo da dati empirici.

$V$ iene mostrata lopportunità di tener conto della non linearità di $1(\xi)$ nelle applicazioni di questa tecnica a taluni problemi interessanti la Geofisica. 


\section{SUMMARY}

Continuing a previous communication the tracks of a-particles emitted by a material laying upon a nuclear emulsion have been theoretically investigated.

Using the general hypothesis that between the range 11 in material and the range $\xi$ in the emulsion for a-particles of given energy exists the relation $\eta=1(\xi)$, the general spectra of the horizontal and vertical projections and the effective lengths have been determined.

The cases in yhich some of the above hypothesis are not verified are discussed.

Numerical calculations are carried out after having made some hypothesis on the form of the function $11=1$, whose constants have been calculated on the basis of the Bethe theory or from empirical data.

It is shoun the advantage of taking in account the non linearity. of 1 (E) in the applications of this tecnique to some problems of interest in Geophysics.

\section{BIBLIOGRAFIA}

(1) D. Palcuro: Ann. di Geof., VI, 1953.

(-) Laviolt-Borsstein: 1, 5, Goltingen, 1952, pg. 365.

(3) J. P. Lonchasir: Journ, de Phys., 14, (1953), 89.

(4) M. G. Maxo: Ann. de Pligs, 11, (1934), 407.

(i) H. A. Bethe - M. S. Livingstont. Rev. of Mod. Phys., 9, (1937), 245.

(6) J. H. П ЕRB: P. R, 7t, (1943), 522.

(7) L. Vicsenon: Jour. de Phys., 14, (1953), 145.

(8) A. Beiser: Rev. of Mod. Phiss., 24, 11952), 273.

(9) M. Joliot - Cinif: Jour. de Phys., 7, (1946), 313.

(10) F. Bachelet - E. M. Ferhetti Sforzivi - F. Ippolito: N. C. VIII, (1951), 851.

(11) Ravkama - Sahama: Geochemistry, Chicago, 1950, pg. $63 t$.

(15) G. Imbò - L. Casertavo: Ann. di Geof., V, 11952), l.

(13) M. Jolot - C.crie: Les radinélements naturels, Paris, 1946.

(14) L. Barbera elc.: Ann. di Geof., VI, (1953). 